\title{
VIOLENCIA EN LA RELACIÓN DE \\ PAREJA DE LOS ALUMNOS DE ENFERMERÍA, 2015
}

\section{VIOLENCE IN THE RELATIONSHIP OF NURSING STUDENTS, 2015}

Recibido 28 de abril 2015 Aceptado 22 de septiembre 2015

Correspondencia:

M. en C. Adela Alba Leonel Antiguo camino a Xochimilco y Viaducto Tlalpan Col. San Lorenzo Huipulco C. P. 14370

Tlalpan, México, D. F. Tel. 55562332 Ext. 269 adelaalbaleonel@yahoo.com.mx

\section{Autores:}

M. en C. Adela Alba Leonel

Mitra. en Ciencias Sociomédicas con énfasis en Epidemiología. Profesor de Carrera Asociado "C", Escuela Nacional de Enfermería y Obstetricia, UNAM

M en C. Julio Hernández Falcón

Mtro. en Investigación de Servicios de Salud Profesor de Carrera Asociado "C", Escuela Nacional de Enfermería y Obstetricia, UNAM

Ing. Joaquín Papaqui Hernández

Ing. en Computación y Especialista en Estadística Aplicada, Instituto Mexicano de Seguridad Social

Ing. Rubén Roa Ponce

Ing. en Computación. Técnico Académico Asociado "A", Escuela Nacional de Enfermería y Obstetricia

Ing. Sirenia Pérez Cabrera

Ing. en Computación. Profesor de Asignatura "A", Escuela Nacional de Enfermería y Obstetricia

Palabras clave: violencia en la relación de pareja, violencia física, violencia emocional, violencia psicológica y violencia sexual

Keywords: violence in the relationship, physical violence, emotional violence, psychological violence and sexual violence 


\section{RESUMEN}

Introducción: La Encuesta Nacional sobre Dinámica de las Relaciones en los Hogares (ENDIREH) 2017, reporto que el $46.1 \%$ de las mujeres de más de 15 años han sufrido violencia de parte de sus parejas.

Objetivo: Conocer y adentrarse en el estudio de violencia en las relaciones de pareja de los estudiantes de la ENEO-UNAM.

Metodología: Se realizó un estudio descriptivo, la unidad de observación fueron alumnos de las carreras: Lic. en Enfermería y Lic. en Enfermería y Obstetricia, el muestreo fue no probabilístico. Se aplicó un cuestionario exprofeso "Detección de violencia en pareja", de 15 reactivos.

Resultados: Se estudiaron 112 alumnos, el 84.8\% correspondió al género femenino, el 51\% tienen menos 20 años, 37\% tiene entre 20-23 y el 12\% más de 23 años de edad. El 85.7\% de las (os) alumnos, informo que tiene violencia emocional (controla su tiempo, celos y la acusa de ser le infiel), El 38.3\% está en proceso de gestación de dependencia, el 15.8\% tiene violencia económica, el 1.8\% dijo tener violencia física, el 8.9\% reportó violencia psicológica y solo se reportó un caso de violación por parte de su pareja.

Conclusiones: Se confirma que existe violencia en las relaciones de pareja de tipo: emocional, dependencia, física, económica y sexual en los alumnos de enfermería, por lo que se debe de implementar y aplicar una intervención para disminuir esta. Es necesario fomentar que toda pareja llegue a acuerdos, donde se establezcan límites y distancias, sobre todo fomentar el mutuo respeto.

Palabras clave: violencia en la relación de pareja, violencia física, violencia emocional, violencia psicológica y violencia sexual.

\section{ABSTRACT}

Introduction: The National Survey on the Dynamic of Household Relations (ENDIREH) 2011, reported that $46.1 \%$ of women over 15 have experienced violence by their partners.

Objective: To determine and enter the study of violence in relationships of students ENEO-UNAM.

Methodology: A descriptive study, the observation unit were students of the races: Lic. Nursing and Nursing and Midwifery, was non-probability sampling. a questionnaire exprofeso "Detection of violence as a couple" of 15 items was applied.

Results: 112 students were studied, $84.8 \%$ were female, $57 \%$ are under 20 years, 37\% are between 20- 23 and $12 \%$ over 23 years old. 85.7\% of the (old) students reported having emotional violence (control your time, jealousy and accuses her of being unfaithful), $38.3 \%$ is in the making of dependence, $15.8 \%$ is economic violence, $1.8 \%$ reported physical violence, $8.9 \%$ reported psychological violence and only one reported case of rape by an intimate partner.

Conclusions: It is confirmed that there is violence in the relationships of type: emotional dependency, physical, economic and sexual in nursing students, so it must implement and apply an intervention to reduce this. It is necessary to encourage all couples reach agreements, which set limits and distances, especially foster mutual respect.

Keywords: violence in the relationship, physical violence, emotional violence, psychological violence and sexual violence. 


\section{INTRODUCCIÓN}

D e acuerdo con los datos de la Encuesta Nacional sobre Dinámica de las Relaciones en los Hogares (ENDIREH) 2017 ? reporta que el $46.1 \%$ de las mujeres de más de 15 años han sufrido violencia de parte de sus parejas. La entidad federativa con mayor prevalencia de violencia de pareja a lo largo de la relación es el Estado de México, con 57.6\%, Nayarit 54.5\%, Sonora 54\%, el Distrito Federal $52.3 \%$ y Colima $51.0 \%$

La violencia de pareja se puede clasificar en emocional, económica, física y sexual de acuerdo con los resultados de la ENDIREH del 2011, donde se reporta que la violencia emocional existe en 4 de cada 10 mujeres en México, el 43.1\% reporto haber sido humilladas, menospreciadas, encerradas, que les han destruido sus pertenencias o enseres del hogar, vigiladas, amenazadas con ser abandonadas, correrlas de la casa o quitarle a sus hijos, amenazadas con algún arma o con matarlas o matarse la pareja.

En cuanto a la violencia económica se reportó que 2 de cada 10 mujeres 24.5\% han recibido reclamos por parte de su pareja por la forma en que gastan el dinero, les han prohibido trabajar o estudiar, o les han quitado dinero o bienes (terrenos, propiedades, etc.).

En lo que respecta a la violencia física, se informó que de cada 100 mujeres 14 han sido golpeadas por su pareja, amarradas, pateadas, tratado de ahorcar o asfixiar, o agredido con un arma.

En el caso de violencia sexual, se reporta que de cada 100 mujeres 7 les han exigido o las han obligado a tener relaciones sexuales con sus propias parejas, sin que ellas quieran, o las han obligado a hacer cosas que no les gustan.

El origen de la violencia se considera puede estar relacionada con la división social del trabajo: hombres y mujeres que se desarrollan en el marco de las sociedades con distintos roles y actividades que contribuyen a generar contradicciones en la vida cotidiana. Michel Foucault2 resalta las oposiciones que se han suscitado entre hombres y mujeres; son luchas transversales que abarcan el grueso de las sociedades, los efectos de la lucha son el poder y no busca al enemigo real sino el más inmediato.

El Instituto Nacional de las mujeres indica que la violencia tiene tres fases: en la primera se acumula tensión, en la segunda ocurre la agresión y en la tercera de la reconciliación o una luna de miel y en esta última el agresor o pareja promete que no volverá a suceder y la víctima lo perdona. Sin embargo a cada momento la amenaza violenta está presente. El caso más alarmante es el de la familia ya que su manifestación es impactante y cruel.

En la perspectiva clásica la violencia puede ser clasificada en dos etapas según la psicología social. Un elemento interno que desencadena la violencia; un instinto latente que frente a los factores sociales y ambientales se expresa. La frustración-agresión reconoce el elemento interno pero solo si existen condiciones externas que propician la frustración.

Una alternativa a la explicación de la violencia es el del aprendizaje social es decir que todo se puede aprender por imitación incluida la violencia 3 . Es claro que la violencia es siempre por alguien y contra alguien. Si no existe relación alguna es claro que la violencia no ocurre. Lo que la ubica en un contexto, le califica de una acción intencional y la consecuencia del acto es la agresión. Un dato más es que la violencia es un acto anti normativo. De manera que la violencia debe colocarse en un marco normativo; en un contexto grupal-social y en sus formas de interacción como lo es la institución del lenguaje. Es el análisis del discurso de esta forma un análisis de la violencia.

Los discursos violentos revelan a las sociedades y sus actores sin lugar a dudas; ellos encierran los valores y sus usos. A la violencia se agrega su carácter simbólico que trasluce en los discursos. La 
deconstrucción de la agresividad y la violencia es un ejercicio necesario ya que el "monstruo" se encuentra oculto en las palabras y su fuerza.

Por su parte Elena Casado en su estudio sobre el análisis crítico sobre los indicadores de violencia ${ }^{4}$ concluyen en la necesidad de aclarar los términos y los contextos de la violencia; si se quiere tener claridad de la violencia y el conflicto.

Cuando la violencia sucede en el hogar el efecto psicológico en las mujeres y los menores dependen de la capacidad de respuesta; sin embargo la naturaleza de la violencia y su persistencia suelen generar efectos inmediatos y a largo plazo. El distrés y estrés postraumático pueden ser la conclusión del malestar. En todo caso la experiencia de los síntomas originados por la violencia involucra la percepción de los propios síntomas 5 .

Un primer indicador para saber que una mujer está inmersa en el círculo de la violencia es tener una sensación de malestar constante o depresión, indica Femat González.

Los cambios en el patrón de sueño, la ansiedad y el nerviosismo; la tristeza y la aflicción; así como asustarse con facilidad suelen reportarse con frecuencia como consecuencias de la violencia domésticá.

Se reconoce que a pesar de que la violencia tiene una elevada prevalencia; su detección es aun pobre y de baja confiabilidad. La detección tardía de la violencia no solo afecta la salud manteniendo la elevada incidencia de enfermedades crónicas?.

Judith Butler en su libro "vida precaria. El poder del duelo y la violencia" reconoce que los acontecimientos ocurridos el 11 de septiembre del 2001 no solo han configurado el escenario político hoy definitorio del sistema democrático. Sino también se propone una nueva reflexión acerca de la dimensión de la vida política y la exposición a la violencia. La vulnerabilidad corporal como condición de la comunidad. La vulnerabilidad como una condición común a todo ser humano que ahora experimenta de manera permanente y al margen de la libertad.

Para Butler ${ }^{8}$, la cuestión no radica solamente en la existencia de discursos deshumanizadores, sino en la falta de reconocimiento que provocan los limites.

El presente estudio tiene la finalidad de conocer y adentrarse en el estudio de la violencia en las relaciones de pareja de sus estudiantes de la ENEO-UNAM.

\section{MATERIAL Y MÉTODOS}

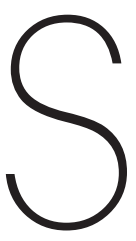

e realizó un estudio cuantitativo, descriptivo, transversal; el universo de estudio fue la Escuela Nacional de Enfermería y Obstetricia de la UNAM, durante el ciclo escolar 2015-2, la unidad de observación fueron alumnos de las carreras de Licenciatura en Enfermería (LE) y Licenciatura en Enfermería y Obstetricia (LEO); el muestreo fue no probabilístico. Se les aplicó un cuestionario exprofeso "Detección de violencia en pareja", de 15 reactivos, los datos fueron recolectados en los meses de febrero y marzo de 2015.

Los criterios de inclusión fueron alumnos de los diferentes semestres de la LE y LEO de la Escuela Nacional de Enfermería y Obstetricia de la UNAM, criterios de exclusión: alumnos que no quisieron participar y criterios de eliminación: que no hayan respondido el cuestionario correctamente.

Se les aplicó un cuestionario el cual contenía datos generales como sexo, edad, y 15 reactivos específicos de violencia con su pareja. El instrumento puede ser aplicado de forma individual o colectiva, sin embargo este se aplicó de forma individual. El tiempo de respuesta fluctuó entre 5 a 8 minutos.

La información se capturó y analizó en Excel, se realizó un análisis descriptivo y relativo para cada variable de la investigación.

\section{4}

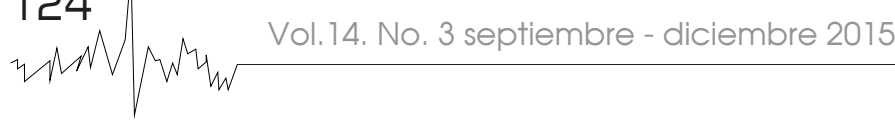




\section{RESULTADOS}

S

e estudiaron 112 alumnos, el 84.8\% correspondió al género femenino y el 15.2\% masculino, el 51\% tienen menos 20 años, el 37\% tiene entre 20- 23 años y el 12\% más de 23 años de edad.

El 86\% de las (os) alumnos de la ENEO, se encuentran en el proceso de indicio hacia posibles malos tratos por parte de su pareja, ya que su pareja continuamente controla su tiempo, es celoso (a) o posesivo (a) y la (o) acusa de ser infiel y coquetear con otros.

El 38\% de la población en estudio se encuentra en proceso de gestación de dependencia con su pareja, ya que está constantemente la desanima a salir o mantener relaciones con amigos (as) y con la familia, así mismo le dice que no quiere que estudie ni trabaje.

En cuanto al proceso de control económico el 15\% de los estudiantes se encuentran en este proceso ya no le permite trabajar y controla sus gastos y los obliga a que le rindan cuentas.
Alumnos de enfermería, que su pareja les controla su tiempo, cela o acusa de ser infiel, 2015

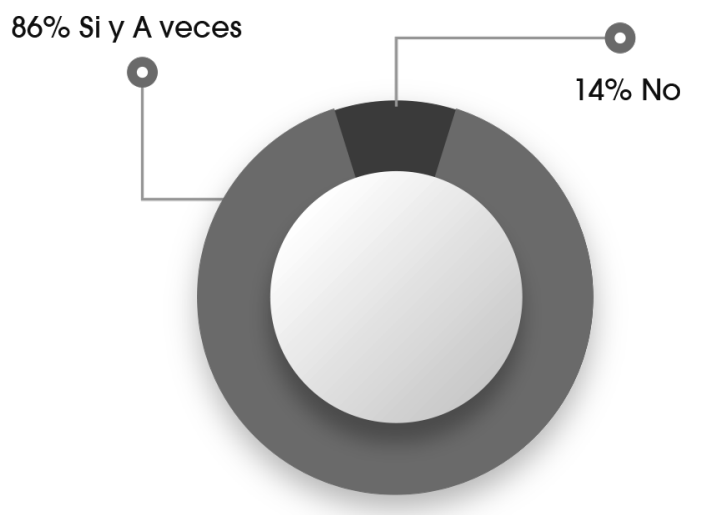

Alumnos de enfermería, que su pareja los desmotiva a salir con amigos, familiares y que no trabajen, 2015

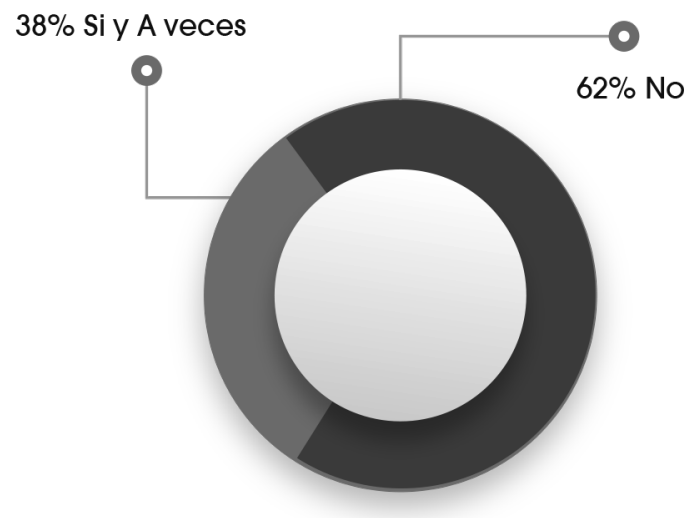

Alumnos de enfermería, que su pareja les controla sus gastos y obliga a rendir cuentas, 2015

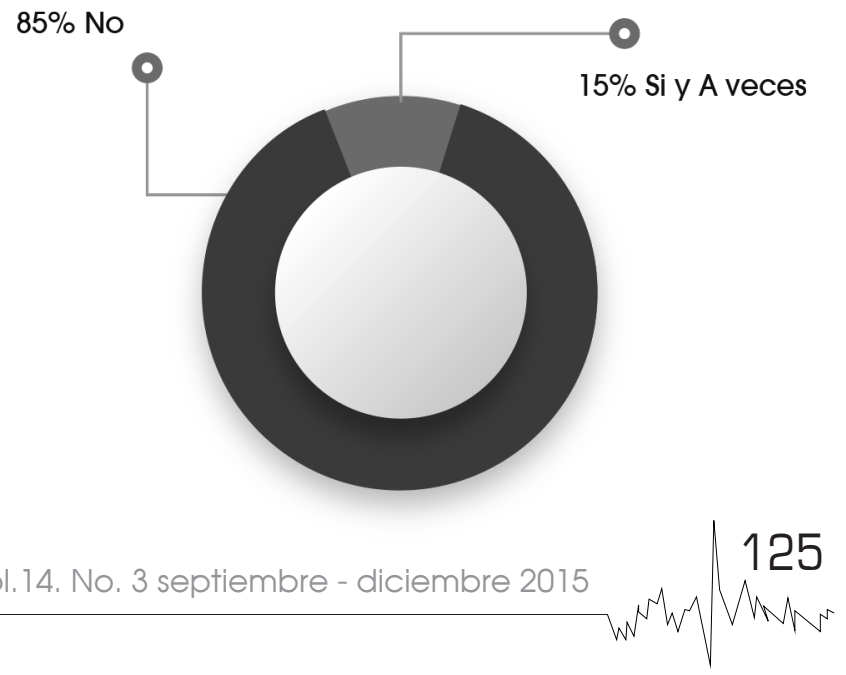


Alumnos de enfermería, que su pareja destruye sus objetos y las amenaza, 2015

El 19\% de la población en estudio se encuentra en proceso de maltrato grave, ya que su pareja la humilla en frente de los demás, rompe y destruye sus objetos con valor sentimental, la amenaza y arremete.

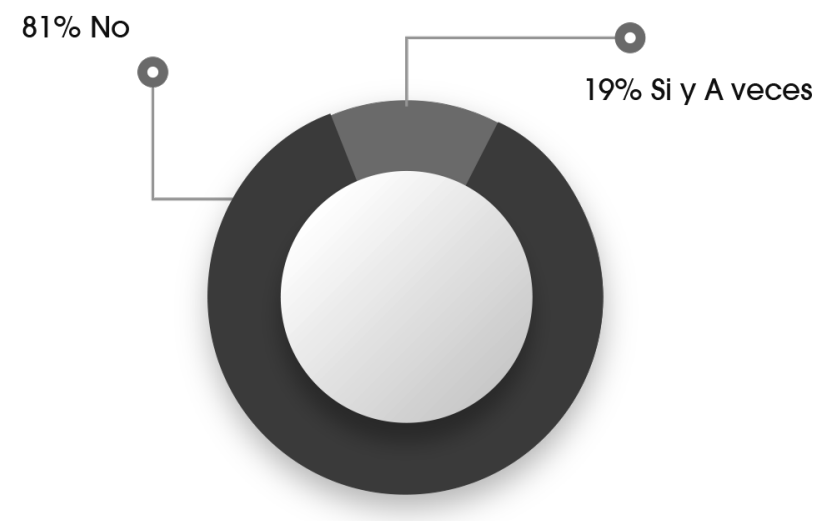

Se encontró un solo caso de violación, ya que la obliga a mantener relaciones sexuales.

El 8.9\% de la población se encuentra en situación de maltrato psicológico/emocional, y castiga en frente de los demás, desautoriza delante de los hijos (as) y compite con ellos por atención.

Distribución de violencia de su pareja, según tipo de violencia en alumnos de la ENEO-UNAM, 2015

Tipo de violencia

Emocional (controla su tiempo, celos y la acusa de ser le infiel)

Emocional de dependencia (la desanima a mantener relaciones con amigos y familiares, que no estudie ni trabaje)

Económica (no quiere que trabaje y controla sus gastos)

Sexual (la obliga a tener relaciones)

Física (llega a empujarte o golpearte)

Psicológica y emocional (la castiga en frente de los demás, me desautoriza delante de los hijos (as) y compite con ellos por mi atención)

\section{Porcentaje}

87.5

38.3

15.2

0.89

1.8

8.9 


\section{DISCUSIÓN}

A ctualmente se sabe que la violencia de género en el noviazgo tiende a crecer, ésta inicia con poner apodos ofensivos a la mujer, darle empujones "en broma, chantajearla, manipularla, celarla e inclusive controlarla, hasta que la mujer disminuya o pierda el autoestima; e iniciar la violencia sexual y finalmente a los golpes?

En la presente investigación se encontró una prevalencia alta de las (os) alumnos (as) donde su pareja continuamente les controla su tiempo, es celoso (a) o posesivo (a) y la (o) acusa de ser infiel y coquetear con otros. El hecho de que el hombre/ mujer tenga interés extremo de saber todo lo que la mujer/hombre hace, deja ver que hay desconfianza o sospecha de que existe infidelidad. La pareja trata de comprobar, inspeccionar, fiscalizar e intervenir en su vida para lograr el dominio sobre la pareja. (Violencia psicológica)

Los celos denotan propiedad y, sin embargo, muchos/ as jóvenes los perciben como muestras de cariño, de interés, preocupación por parte de la pareja, incluso hay quienes se sienten halagados/as porque su pareja los/as cela, y se escuchan dichos como "me cela porque me quiere". 10

Estos datos concuerdan con lo publicado por el Instituto Politécnico Nacional (IPN) en su estudio Relaciones de pareja que establecen estudiantes ${ }^{10}$, sin embargo la prevalencia en nuestro estudio es más alta quizás porque esta profesión es inminentemente femenina, donde por cuestiones sociales se deriva a los llamados roles de género, donde se marca estereotipos y condiciona las funciones o papeles de ambos sexos, limitando así las potencialidades de cada persona ${ }^{17,12}$. Donde se establece que las mujeres, deben ser emotivas, débiles, dulces, asustadizas, dependientes, sumisas, subordinadas a la autoridad masculina.

La violencia física puede iniciar con ligeros golpecitos en forma de juego, palmaditas, pequeños empu- jones, cachetadas-caricias, pellizcos en forma de broma, casi siempre sin dejar huella, sin embargo posteriormente puede ir avanzando hasta, que ésta puede ser evidente por el daño o lesiones que puede producir de forma interna, externa o bien en ambas, en el cuerpo de la persona. Aun cuando la prevalencia no es alta existe este tipo de violencia en los (as) alumnos (as). Es de llamar la atención que las lesiones internas generalmente se diagnostican mucho tiempo después, de que se ocasionaron pudiendo inclusive provocar la muerte ${ }^{13,14}$.

Aun cuando la violencia sexual es casi nula esta existe en la población estudiantil, cabe señalar que esta no se compara con los datos reportados en la ENDIREH.

Como bien se menciona en el estudio de Relaciones de pareja que establecen estudiantes del IPN. "La violencia atenta contra la vida, la libertad, la salud, la seguridad, el desarrollo psicoemocional, la sexualidad, la dignidad y la paz; es decir, lesiona los derechos inherentes de las personas y muestra con nitidez la opresión de género", por lo que es necesario trabajar y en la medida de lo posible erradicar este problema.

\section{CONCLUSIONES}

a violencia en las relaciones de pareja que los/las jóvenes entablan es una situación con rasgos alarmantes. Como bien sabemos es imposible pensar que las relaciones de L pareja sea siempre todo amor y dulzura. Sin embargo es importante señalar que también se generan conflictos, debido a que las personas son diferentes en cuanto a intereses, deseos, gustos, valores, creencias, actitudes, entre otras. Por lo que cuando se establece una relación de pareja es posible que se presenten algunos conflictos, sin embargo estos se pueden resolver a través del dialogo, conciliación y negociación tratando siempre de mantener el equilibrio entre las parejas. 
Es necesario fomentar que toda pareja llegue a acuerdos, donde se establezcan límites y distancias, sobre todo fomentar el mutuo respeto. Para así evitar el uso de autoridad, poder y fuerza que conduzcan a la violencia de pareja ${ }^{15,16}$

Detener la violencia desde sus inicios es importante para no dar lugar a que aumenten los niveles de violencia y sobre todo no perder en cuenta la situación que se vive, porque de no ser así se puede terminar en desenlaces fatales como el homicidio o suicidio.

Los resultados de la presente investigación confirman que la violencia en las relaciones de su pareja constituye un problema, por lo que se debe de aplicar una intervención oportuna para prevenir y disminuir este.

Las estrategias de prevención incluyen la elaboración de un código de ética, el dar mayor participación y protagonismo a los jóvenes involucrados. Atender de manera específica a las víctimas y los agresores de manera que se encuentren caminos constructivos de las relaciones.

\section{REFERENCIAS BIBLIOGRÁFICAS}

1. Fuente de la información 2011: INMUJERES con base en la Encuesta Nacional sobre la Dinámica de las Relaciones en los Hogares (ENDIREH) 2011. Bases de datos publicadas el 29 de julio de 2013. ajustadas a las Proyecciones de la Población en México que el Consejo Nacional de Población actualizó el 16 de abril de 2013

2. Foucault Michel. El sujeto y el poder. México: Revista Mexicana de Sociología V.50 n.3 1988: 3-20. Disponible en: http://terceridad. net/wordpress/wp-content/uploads/2011/10/Foucault-M.-El-sujeto-yel-poder.pdf (15 abril 2015)

3. Domenech I Argemi M, Iñiguez Rueda L. La construcción social de la violencia. Barcelona: Universidad Autónoma de Barcelona Athenea Digital n.2 2002. Disponible en: http://www.raco.cat/index.php/Athenea/article/viewFile/34105/33944 (14 abril 2015)

4. Casado Aparicio Elena. Análisis crítico de los indicadores de violencia de género en parejas heterosexuales en España. http://www.redalyc. org/articulo. oa?id=297124737008

6. Canaval Gladys E, Cecilia González M, Humphreys J, De León N, González N. Violencia de pareja y salud de las mujeres que consultan a las comisarías de familia, Cali Colombia. Colombia: Universidad de Antioquia v.17 n.2 2009. Disponible en: http://www.scielo.org.co/ pdf/iee/v27n2/v27n2a06 (13 abril 2015)

6. SSa. Violencia contra las mujeres: un problema de salud pública. México: SSa- Centro nacional de Equidad de Género y Salud Reproductiva. Disponible en : http://www.inegi.org.mx/inegi/contenidos/espanol/eventos/vigenero/dia29/panel5_mesas/Violencia/ Violencia-usuarias-serv_salud.pdf (14 abril 2015)

7. Arredondo-Provecho AB, Broco B.M, Alcalá Ponce de LT, Rivera Álvarez A, Jiménez TI, Gallardo-Pino C. Profesionales de atención primaria de Madrid y violencia de pareja hacia la mujer en el año 2010. España: Rev Salud Publica 2012. 86;85-96. Disponible en:http:// www.msssi.gob.es/biblioPublic/publicaciones/recursos_propios/resp/ revista_cdrom/vol86/vol86_7/RS861C_85.pdf (20 marzo 2015)

8. Buther Judith. Vida Precaria. Argentina: Editorial Paidós 2006.

9. Nieto Mitsi, "Aguas con la violencia en el noviazgo", extraído de: $h$ ttp://www.terra.com. $m x /$ articulo. aspx?articuloid=20406\&spaginaid= 7 (27 marzo 2015)

10. IPN. Género y amor: Principales aliados de la violencia en las relaciones de pareja que establecen estudiantes del IPN, 2012, 1-30.

11. Bustos, Olga. La formación del género: el impacto de la socialización a través de la educación. México: Consejo Nacional de Población, 1998

12. Lagarde, Marcela. Democracia genérica. México: RepenMéxico, 1994

13. Torres Falcón, Marta. Al cerrar la puerta. Amistad, amor y violencia en la familia. México: Editorial Norma, 2005

14. Martínez, María Concepción, "Violencia familiar desde la perspectiva de género". En Jóvenes por una vida sin violencia. Centro de Apoyo a la Mujer. México: "Margarita Magón" A. C., 2004.

16. Corsi, Jorge. Violencia Familiar: una mirada abarcativa sobre un grave problema social. Paidós: Buenos Aires, 1994

16. Olivares, Samantha. Resolución no violenta de conlictos. México: Centro de Apoyo a la Mujer "Margarita Magón" A. C., 2004.
128 भMN $\sqrt{4} h_{w}$ Vol. 14. No. 3 septiembre - diciembre 2015 